\title{
Restricciones al consumo de alcohol en un grupo de adolescentes mexicanos
}

\author{
Restrictions on Alcohol Consumption in a Group of Mexican Adolescents ${ }^{1}$
}

\author{
Sarahí Alanís Navarro* \\ Shoshana Berenzón Gorn** \\ Fernando Vázquez Pineda* \\ Fernando A. Wagner Echeagaray*** \\ Georgina Cárdenas López* \\ * universidad nacional autónoma de MÉxICO \\ * * instituto nacional de psiquiatría \\ *** MORGAN STATE UNIVERSITY
}

\section{RESUMEN}

El objetivo del estudio fue identificar las restricciones al consumo de alcohol en un grupo de adolescentes mexicanos. Participaron 90 estudiantes (54\% mujeres y 46\%, hombres) de 14 a 16 años. Los datos se recopilaron mediante la técnica de redes semánticas naturales modificadas. Las frases utilizadas para explorar la disponibilidad de la sustancia y las restricciones a la conducta de consumo fueron: 1) Los adolescentes pueden conseguir alcohol en... y 2) Es difícil beber cuando... Los tres primeros reactivos del Alcohol Use Disorders Identification Test se utilizaron para identificar la ingesta de alto riesgo $(\geq 3)$. Se observó que los hombres con consumo de alto riesgo reportaron un mayor número de medios para conseguir alcohol. En contraste, las mujeres con consumo de bajo riesgo identifican una mayor cantidad de restricciones. Los resultados pueden contribuir a fortalecer los programas preventivos al mostrar variables que incrementan la dificultad para que los adolescentes consuman alcohol.

Palabras clave: consumo de alcohol, adolescentes, economía conductual, restricciones, prevención.

\section{ABSTRACT}

The study aimed to identify the restrictions on alcohol consumption in a group of Mexican adolescents, with 90 students taking part (54\% women and 46\% men) aged between 14 and 16. The data were compiled using the technique of modified natural semantic networks. The phrases used to assess the availability of the substance and the restrictions on consumption were: 1) Adolescents can obtain alcohol from... and 2) It's difficult to drink when... The first three questions of the Alcohol Use Disorders Identification Test were used to identify high-risk consumption ( $\geq 3$ ). It was found that men with a high-risk of consumption reported more ways of obtaining alcohol. Women, on the other hand, with low-risk consumption, identified more restrictions on consumption. The results can help strengthen prevention programs, by showing variables that make it harder for adolescents to consume alcohol.

Keywords: alcohol consumption, adolescents, behavioral economics, restrictions, prevention

Fecha de recepción: 5 de diciembre de 2014 Fecha de aceptación: 30 de mayo de 2015

Para correspondencia: Sarahí Alanís Navarro, alaniszarah@gmail.com

${ }^{1}$ El estudio fue realizado gracias al apoyo del Conacyt y del Programa de Maestría y Doctorado en Psicología de la Universidad Nacional Autónoma de México. 
En México el alcohol está presente en diversos contextos sociales. El consumo de esta sustancia es ampliamente aceptado, incluso entre los adolescentes, quienes en las encuestas nacionales de adicciones representan al grupo con mayor probabilidad de progresar al abuso y a la dependencia. En este sector de la población $17.3 \%$ de los hombres y $11.7 \%$ de las mujeres reportan un consumo alto (Medina-Mora et al., 2012), patrón caracterizado por la ingesta de cinco copas o más por ocasión, entre los hombres, y por cuatro o más, entre las mujeres. Es importante señalar que la mayor proporción de la carga de enfermedad asociada con el uso de alcohol es atribuida a este patrón de consumo (World Health Organization, 2011).

Si bien los adolescentes constituyen el grupo más sano, el que utiliza menos servicios de salud y donde se registran menos defunciones (Celis, 2003), las primeras causas de muerte y los problemas que motivan el uso de servicios de urgencias y de salud mental se relacionan con el consumo de drogas, en particular de alcohol (Borges, Benjet, Medina-Mora, Orozco \& Wang, 2008; Celis, 2003; Guerrero, Muñoz, Sáenz, Pérez \& Reynales, 2013). El escenario en torno a la ingesta de alcohol en los adolescentes muestra la necesidad de incrementar los esfuerzos para disminuir esta problemática.

En diversos países, incluyendo México, las políticas públicas que han mostrado ser más efectivas para reducir el consumo de alcohol y los problemas asociados a él tienen una estrategia común: incrementar las restricciones a esta sustancia (Babor et al., 2010; Medina-Mora, Robles, Cortina \& Real, 2009; Toumbourou et al., 2007). Algunos ejemplos de estas medidas son el aumento a los impuestos al alcohol, la restricción de los horarios, días y puntos de venta, así como las sanciones por manejar en estado de ebriedad.

Por un lado, el problema que representa el consumo de alcohol en la población adolescente y, por otro, la efectividad de las estrategias dirigidas a incrementar las restricciones a éste, motivaron el presente estudio. El objetivo de la investigación es identificar las restricciones al consumo de alcohol en un grupo de adolescentes mexicanos.

El trabajo se enmarca en los modelos de la economía conductual, en los cuales la dependencia hacia una droga puede estudiarse a partir de la demanda por su consumo, en función de las variables del ambiente que la regulan (DeGrandpre \& Bickel, 1996). De esta manera, existen variables que aumentan la demanda y otras que la reducen.

Los estudios de economía conductual dirigen la atención hacia cómo emergen, se desarrollan y cambian los patrones de uso y abuso de sustancias en función de variables del contexto ambiental (Vuchinich \& Heather, 2003). Las investigaciones se centran en contestar tres preguntas: 1) ¿cómo varía el uso de la sustancia con los cambios en las restricciones directas sobre el acceso a la sustancia (por ejemplo, con los cambios de precio o la reducción en la disponibilidad de la sustancia)?; los estudios de este tipo manipulan las restricciones directas al acceso a la sustancia y registran cambios en el consumo, manteniendo constante el acceso a otras actividades (DeGrandpre, Bickel, Hughes, Layng \& Badger, 1993); 2) ¿cómo cambia el uso de la sustancia a partir de la modificación en las restricciones de otras actividades valiosas (trabajo, ocio, actividad social)?; en estos trabajos se miden las variaciones en el consumo derivadas de la manipulación de las restricciones a las actividades alternativas valiosas, mientras el acceso a la sustancia se mantiene constante (Vuchinich \& Tucker, 1988);3) ¿cómo cambia la distribución de la conducta cuando los consumidores eligen entre resultados que varían en cantidad y demora?; en esta línea de investigación el consumo depende de la cantidad de recompensa y de la demora para obtenerla, cuando las actividades están disponibles simultáneamente (Murphy, Correia \& Barnett, 2007; Bickel \& Marsch, 2001).

El presente estudio se basa en la primera de las líneas de investigación citadas: ¿cómo varía el uso de la sustancia con los cambios en las restricciones directas sobre el acceso a ella? Una de las principales estrategias para incrementar las restricciones directas al acceso a la sustancia es manipulando el precio o costo. El incremento en el precio está asociado a una disminución en el consumo, principio que se describe en una función de demanda (DeGrandpre \& Bickel, 1996). Es importante destacar que en los modelos de economía conductual el costo hace referencia no sólo al monetario, sino también al temporal y conductual (Murphy et al., 2007). El costo temporal involucra el tiempo invertido por el individuo para consumir, incluyendo el que destina a conseguir la sustancia y el que invierte en recuperarse 
de sus efectos adversos. El costo conductual es el esfuerzo requerido para adquirir la sustancia, así como las consecuencias legales, sociales o de salud derivadas del consumo.

Entre las técnicas utilizadas actualmente para estudiar la relación entre el costo monetario y la demanda de consumo destacan las tareas de compras hipotéticas. En éstas, los participantes reportan la ingesta de una sustancia a diferentes precios. El procedimiento ha mostrado ser confiable debido a la correspondencia entre la compra hipotética y el consumo real de la sustancia (Amlung, Acker, Stojek, Murphy \& MacKillop, 2012). Al emplear estas tareas en un grupo de estudiantes consumidores de alcohol se observó que la demanda por la sustancia se mantuvo en un primer rango de precios, pero disminuyó ante su incremento (Murphy \& MacKillop, 2006).

La demanda por la sustancia también varía en función de otras restricciones ambientales, entre ellas el costo conductual y la disponibilidad de tiempo. En la población estudiantil una de las variables ambientales analizadas con mayor frecuencia son los requerimientos escolares. Se ha observado que los estudiantes reportan un menor consumo de alcohol cuando tienen un examen o clases al día siguiente (Del Boca, Darkes, Greenbaum \& Goldman, 2004; Gentile, Librizzi \& Martinetti, 2012; Skidmore \& Murphy, 2011) y el efecto es mayor si las responsabilidades académicas son antes de las diez de la mañana (Wood, Sher \& Rutledge, 2007). En contraste, las restricciones son menores los fines de semana y días festivos, periodos donde se registra una mayor proporción de episodios de consumo (Del Boca et al., 2004; Tremblay et al., 2010; Rodríguez, Angulló \& Angulló, 2003).

A partir de estas observaciones, algunos investigadores consideran que una estrategia para elevar el costo de la conducta de consumo es incrementar las demandas académicas, como las horas de clase obligatorias de estudio y de prácticas o servicio social (Borsari, Murphy \& Barnett, 2007), así como promover la participación de los estudiantes en el trabajo voluntario (Weitzman \& Kawachi, 2000). Murphy et al. (2007) plantean que al incrementar las demandas académicas el presupuesto de tiempo disponible disminuye (al menos en relación con el tiempo libre) y el costo personal de la conducta aumenta, haciendo más difícil el consumo excesivo sin afectar el cumplimiento de las demandas escolares. Por ejemplo, experimentar una resaca puede tener un costo mayor si el estudiante tiene una responsabilidad al día siguiente, como un examen, en comparación con un escenario donde no tiene una actividad por la mañana y puede dormir sin experimentar consecuencias negativas.

La evidencia sobre el papel del costo monetario, temporal y conductual, como restricciones directas de la demanda de ingesta de alcohol es amplia y robusta. Sin embargo, para promover en nuestro país estrategias dirigidas a elevar el costo de la conducta de consumo en los adolescentes, primero es necesario identificar cuáles son algunas de las restricciones a él en el entorno inmediato de los adolescentes mexicanos. En este caso, se recopila información de un grupo de estudiantes que asisten regularmente a la escuela en una zona considerada de alto riesgo para el consumo de drogas, con base en los criterios definidos por el Estudio Básico de Comunidad Objetivo (EвCO, Centros de Integración Juvenil, 2007).

\section{MÉTODO}

\section{Participantes}

La muestra intencional, no probabilística, estuvo conformada por 90 estudiantes de dos escuelas públicas ubicadas en comunidades urbanas del Estado de Morelos. Las mujeres representaron 54\% de la muestra los hombres $46 \%$. Las edades fluctuaron entre 14 y 16 años, con un promedio de 14.6 años (S. D. = 0.91).

\section{Instrumentos}

Redes semánticas naturales modificadas. La técnica consiste en una serie de frases incompletas dirigidas a explorar los significados y los conocimientos, socialmente compartidos, en torno a un concepto (Figueroa, González \& Solís, 1981; Reyes-Lagunes \& Valdez, 1993). Los participantes deben completar cada frase con un mínimo de cinco palabras, llamadas definidoras, y jerarquizarlas, con base en la importancia que tienen en la definición del concepto. El número 1 se asigna a la palabra que define mejor el concepto, el 2 a la siguiente, y así sucesivamente. En el estudio se utilizaron dos frases: 1) Los adolescentes pueden conseguir alcohol en... y 2) Es difícil beber cuando... La primera 
explora la disponibilidad de la sustancia; la segunda, las restricciones a la conducta de consumo. La elección de la técnica se sustenta, principalmente, en el carácter social que tiene la ingesta de alcohol entre los adolescentes.

Alcohol Use Disorders Identification Test (AUDIT, Saunders, Aasland, Babor, De la Fuente \& Grant, 1993, adaptado en México por Medina-Mora, Carreño \& De la Fuente, 1998). Es un instrumento desarrollado por la Organización Mundial de la Salud (oms) para identificar el consumo riesgoso de alcohol. El AUDIT está integrado por diez reactivos que representan tres dominios conceptuales: nivel de consumo, dependencia y problemas relacionados. Las opciones de respuesta se presentan en una escala que va de cero a cuatro puntos.

El dominio nivel de consumo está integrado por tres reactivos que miden: 1) la frecuencia de días, 2) el número de tragos por ocasión y 3) la frecuencia con un consumo de más de cinco tragos. Chung, Colby, Barnett y Monti (2002) establecieron que un punto de corte de tres en este nivel es una forma efectiva para identificar el consumo de alto riesgo entre los adolescentes. En jóvenes mexicanos este puntaje también ha mostrado mayor sensibilidad para identificar dicho nivel de consumo (Díaz et al., 2009). En el presente estudio se utilizó el AUDIT para diferenciar a los participantes con base en su patrón de consumo; por ello, sólo se incluyeron los tres primeros reactivos y se estableció un punto de corte de tres para definir el consumo de alto riesgo.

\section{Procedimiento}

Las escuelas se ubicaron en zonas urbanas del estado de Morelos, consideradas de alto riesgo para el consumo. Se eligieron al azar con base en la lista del eвCo (Centros de Integración Juvenil, 2007). La solicitud de apoyo a los directivos se realizó de manera verbal. Los padres de los alumnos que aceptaron participar firmaron un formato donde se describió el objetivo del estudio, la dinámica de la actividad y el carácter confidencial de la información. Las respuestas al cuestionario fueron anónimas. La aplicación de la técnica se llevó a cabo en una sola sesión, dentro de los salones de clase, con grupos intactos.
Los estudiantes respondieron el instrumento que incluía un apartado con instrucciones generales, las dos frases estímulo utilizadas para obtener las redes semánticas, un apartado de datos generales (sexo, edad y escolaridad) y los tres primeros reactivos del AUDIT que exploran el patrón de consumo de alcohol. Los alumnos completaron la tarea en un promedio de 40 minutos. Al término de la actividad se agradeció a los participantes por su colaboración, así como a los profesores y las autoridades de la escuela por su apoyo para realizar el estudio.

Las palabras proporcionadas por los sujetos para definir cada frase se agruparon según el nivel de riesgo y el sexo de los participantes. El análisis se realizó mediante el procedimiento propuesto por Reyes-Lagunes \& Valdez (1993). Para cada grupo se obtuvieron los siguientes valores: 1) tamaño de la red, que indica el número total de definidoras proporcionadas por los sujetos; 2) peso semántico, obtenido al multiplicar la ponderación de las definidoras (la primera tienen un valor de 10, la segunda de 9, y así sucesivamente) por su frecuencia; 3) núcleo de la red, constituido por las definidoras con peso mayor semántico, y 4) distancia semántica cuantitativa, puntaje que señala la distancia entre los elementos de la red. Este último valor se genera mediante una regla de tres, donde a la definidora con mayor peso se le asigna $100 \%$. Las palabras que conforman el núcleo de la red se seleccionan por inspección visual, mediante una gráfica que presenta los pesos semánticos en orden descendente. Las definidoras que se encuentran por arriba de la asíntota (el punto donde la curva se vuelve plana) constituyen el núcleo de la red.

\section{RESULTADOS}

El $46.9 \%$ de los hombres y $34 \%$ de la mujeres obtuvieron un puntaje igual o mayor a tres (consumo de alto riesgo) en los reactivos del dominio nivel de consumo del Audit (vid. tabla 1).

Las palabras que definen los conceptos explorados se muestran según el sexo y el nivel de riesgo de los participantes. En las tablas 2 y 3 se reporta su peso semántico (PS) y distancia semántica cuantitativa (DSC). Este último valor sólo se obtiene para las definidoras que constituyen el núcleo de la red (NR) y en las cuales se centra el análisis. 
Tabla 1. Proporción de hombres y mujeres con consumo de alcohol de alto riesgo

\begin{tabular}{c|c|c|c} 
& Hombres & Mujeres & Total \\
\cline { 2 - 4 } & $(\mathrm{n}=49)$ & $(\mathrm{n}=41)$ & $(\mathrm{N}=90)$ \\
\hline Consumo alto riesgo & $46.9 \%$ & $34.0 \%$ & $41 \%$ \\
\hline Total & $54.0 \%$ & $46.0 \%$ & $100 \%$
\end{tabular}

Tabla 2. Definidoras obtenidas para el concepto: disponibilidad

\begin{tabular}{|c|c|c|c|c|c|c|c|c|c|c|c|}
\hline $\begin{array}{l}\text { Mujeres } \\
\text { alto riesgo } \\
\text { definidoras }\end{array}$ & ps & dsc & $\begin{array}{l}\text { Bajo riesgo } \\
\text { definidoras }\end{array}$ & ps & dsc & $\begin{array}{l}\text { Hombres } \\
\text { alto riesgo } \\
\text { definidoras }\end{array}$ & ps & dsc & $\begin{array}{l}\text { Bajo riesgo } \\
\text { definidoras }\end{array}$ & ps & dsc \\
\hline Tienda & 79 & 100 & Tienda & 147 & 100 & Tiendas & 154 & 100 & Tienda & 185 & 100 \\
\hline Fiestas & 65 & 82 & Fiestas & 135 & 92 & Fiestas & 98 & 64 & Fiestas & 116 & 63 \\
\hline Casa & 39 & 49 & Amigos & 86 & 59 & Oxxo & 83 & 54 & Antro/bar & 89 & 48 \\
\hline Amigos & 34 & 43 & Súper & 45 & 31 & Antro/bar & 80 & 52 & Casa & 86 & 46 \\
\hline Familia & 23 & 29 & Antro/bar & 33 & 22 & Amigos & 58 & 38 & Súper & 61 & 33 \\
\hline Calle & 17 & 22 & Casa & 33 & 22 & Súper & 57 & 37 & Billar & 31 & \\
\hline Súper & 17 & 22 & Oxxo & 23 & & Familia & 45 & 29 & Calle & 27 & \\
\hline Oxxo & 10 & & Familia & 21 & & Casa & 41 & 27 & Oxxo & 23 & \\
\hline Vinatería & 7 & & Escuela & 11 & & Billar & 40 & 26 & Familia & 9 & \\
\hline Billar & 6 & & Calle & 8 & & Calle & 25 & & & & \\
\hline Antro/bar & 6 & & Billar & 5 & & Vinatería & 10 & & & & \\
\hline & & & & & & Escuela & 9 & & & & \\
\hline & & & & & & $\begin{array}{l}\text { Expendio de } \\
\text { cerveza }\end{array}$ & 8 & & & & \\
\hline & & & & & & Feria & 8 & & & & \\
\hline & & & & & & Concierto & 6 & & & & \\
\hline
\end{tabular}

Nota: se reporta el peso semántico (PS) y la distancia semántica cuantitativa (DSC) de cada definidora. 
Tabla 3. Definidoras obtenidas para el concepto: restricciones

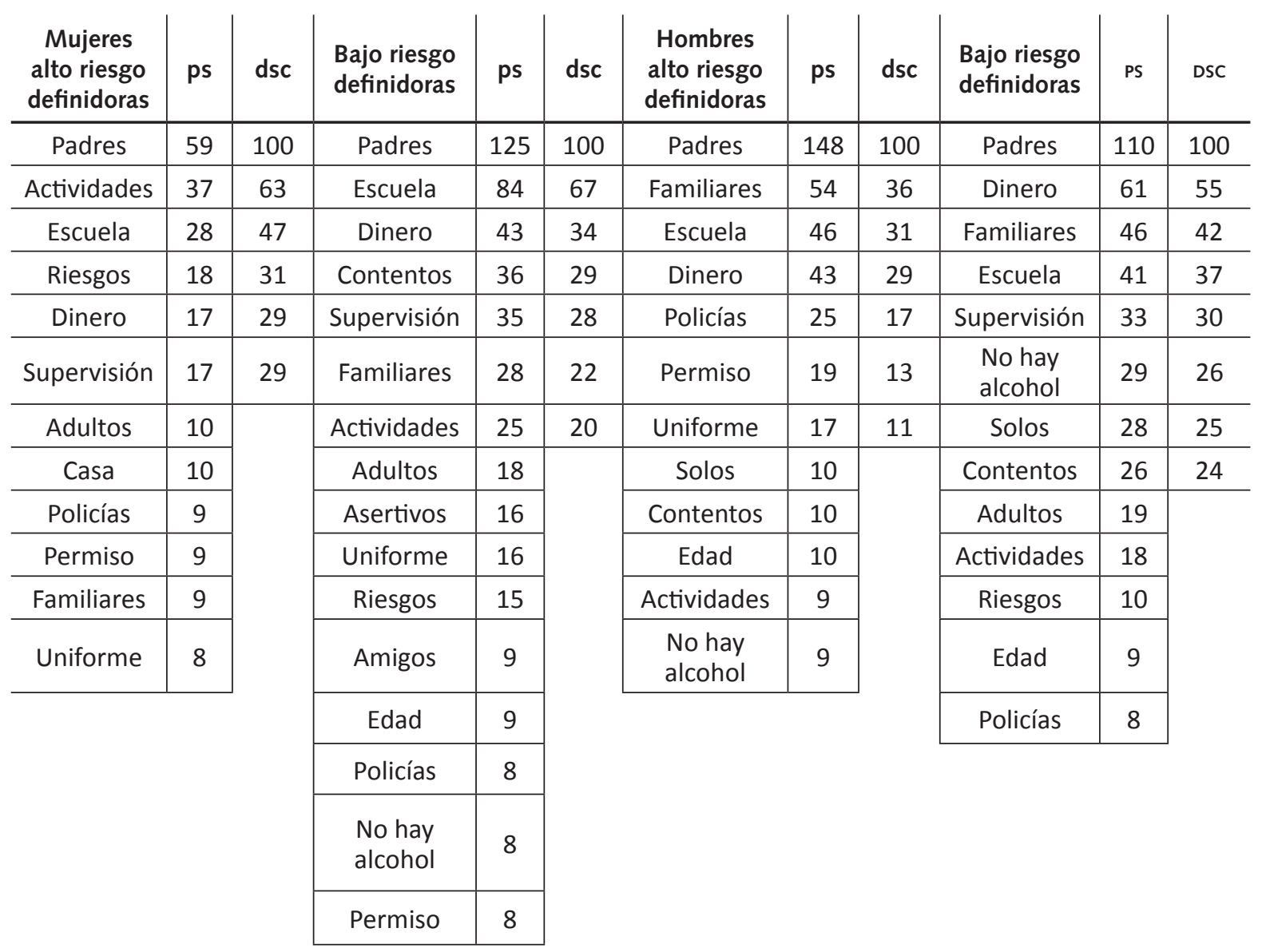

Nota: se reporta el peso semántico (PS) y la distancia semántica cuantitativa (DSC) de cada definidora.

La primera frase estímulo (Los adolescentes pueden conseguir alcohol en...) permitió identificar los lugares y las situaciones donde los adolescentes pueden conseguir alcohol. El tamaño de la red -valor que indica la riqueza semántica del concepto- fue mayor entre los hombres con consumo de alto riesgo. El resultado muestra que estos participantes identifican un mayor número de alternativas para conseguir la sustancia. Las definidoras que se registran sólo en este grupo hacen referencia a establecimientos mercantiles dedicados exclusivamente a la venta de alcohol (vinaterías y expendios de cerveza) y a eventos masivos asociados con la ingesta de la sustancia (conciertos y ferias).

En los cuatro grupos la palabra con mayor peso semántico fue tienda; la segunda, fiestas. Las definidoras obtenidas para el concepto de disponibilidad se clasificaron en dos grupos: 1) medios para adquirir el alcohol (comprándolo y por invitación) y 2) lugares de consumo (públicos y privados). Los adolescentes de la muestra compran el alcohol principalmente en pequeños establecimientos comerciales (tienda), seguido de los grandes (súper). El Oxxo, una cadena comercial de mediana dimensión, fue mencionado en todos los grupos, pero sólo se observa en el núcleo de la red de los hombres con consumo de alto riesgo. Los amigos, como medio para conseguir el alcohol, aparecen en el núcleo de la red de todos los grupos, con excepción de los hombres con consumo de bajo riesgo, mientras la familia está en ambos grupos con consumo de alto riesgo.

En relación con los lugares de consumo públicos la definidora con mayor peso semántico es fiestas; el antro o bar tiene menor peso y no figura en el núcleo de la red de las mujeres con consumo de alto riesgo. En este grupo, la calle permanece en el núcleo de la red; 
mientras el billar está entre los hombres con consumo de alto riesgo. La casa, clasificado como un lugar privado donde los adolescentes pueden conseguir alcohol, es una definidora representativa en todos los grupos.

La segunda frase estímulo (Es difícil beber cuando...) tuvo por objetivo explorar los factores que restringen directamente el consumo de alcohol y que, por lo tanto, pueden contribuir a incrementar el costo de esta conducta. Las mujeres con consumo de bajo riesgo proporcionaron el mayor número de definidoras para este concepto, sugiriendo que ellas identifican un mayor número de restricciones para consumir alcohol.

La definidora con el mayor peso semántico en todos los grupos es la presencia de los padres, seguida de estar en la escuela y no tener dinero. Los significados que otorgaron los participantes al concepto de restricciones se clasificaron en cuatro grupos: 1) individuales, 2) familiares, 3) sociales y 4) costo monetario. Entre las restricciones individuales se destacan estados de ánimo positivos como estar contento, la percepción de riesgo y la asertividad. Estas restricciones aparecen en ambos grupos con consumo de bajo riesgo, aunque sólo estar contentos figura en el núcleo de la red.

Las restricciones familiares más representativas son la presencia de los padres, la supervisión (excepto para los hombres con consumo de alto riesgo), la figura de un familiar y no tener permiso, o estar castigado, definidora registrada en el núcleo de la red de los hombres con consumo de alto riesgo. Las restricciones sociales más representativas son estar en la escuela, portar uniforme y la presencia de policías. Ser menor de edad, considerada como una restricción social, aparece en todos los grupos pero no forma parte de su núcleo de la red. El dinero, clasificado como una restricción monetaria, es una definidora con elevado peso semántico en los cuatro grupos, principalmente entre los participantes con consumo de bajo riesgo.

De manera general, se observaron diferencias entre los grupos de consumo de alcohol de bajo y alto riesgo, respecto a los valores obtenidos mediante la técnica de redes semánticas (tamaño de la red, peso y distancia semántica y núcleo de la red).

\section{DISCUSIÓN}

Las palabras que conformaron el núcleo de la red de los grupos con consumo de bajo y alto riesgo brindan infor- mación útil sobre los recursos, lugares, medios y situaciones que pueden incrementar el costo de la conducta de consumo de alcohol, en los adolescentes de la muestra. En este estudio se observó que el número de situaciones y recursos considerados como medios para conseguir alcohol fue mayor entre los hombres con consumo de alto riesgo, mientras que las mujeres con consumo de bajo riesgo identificaron una mayor cantidad de restricciones.

Lo anterior es consistente con las premisas de la economía conductual, enfocadas en modelar la demanda por el consumo y sus restricciones directas e indirectas (Vuchinich \& Heather, 2003): menos restricciones directas en los consumidores de alto riesgo y más restricciones en los de bajo riesgo.

Es pertinente señalar algunas diferencias entre estos resultados con la finalidad de aportar información dirigida a incrementar restricciones en el entorno inmediato de los adolescentes. Por ejemplo, la familia como recurso para conseguir la sustancia sólo se registra en el núcleo de la red de los participantes con consumo de alto riesgo. Este resultado muestra la relevancia de incrementar las normas familiares hacia el consumo de alcohol, por ejemplo, mediante la supervisión, definidora que aparece como una restricción importante (excepto entre los hombres con consumo de mayor riesgo).

Aunado a la identificación de algunas variables que pueden incrementar el costo del consumo de alcohol entre los adolescentes, los hallazgos del trabajo permiten distinguir contextos que sugieren un costo bajo de la conducta de consumo y, por lo tanto, facilitan el abuso de esta sustancia. Un ejemplo de estos entornos son las fiestas, donde las personas acuden como invitados y el costo monetario para adquirir el alcohol es cero. En el estudio estas situaciones de consumo figuraron como el principal medio para conseguir alcohol, dato que coincide con los resultados obtenidos en otros países (Harrison, Fulkerson \& Park, 2000; Paschall, Grube, Black \& Ringwalt, 2007; Scoppetta, Pérez \& Lanziano, 2011).

Otro escenario que disminuye el costo del consumo es la elevada densidad de establecimientos comerciales que venden alcohol a menores, variable que ha mostrado tener una correlación positiva con el consumo de alcohol y los problemas asociados (Kypri, Bell, 
Hay \& Baxter, 2008). En este trabajo, los participantes mencionaron diversos establecimientos donde consideran posible conseguir alcohol, algunos de los cuales se caracterizan por su elevada densidad (por ejemplo, Oxxo) y, por lo tanto, reducen las restricciones al acceso para adquirir la sustancia.

En contraste con la información anterior, el costo monetario (dinero) figuró entre las definidoras con mayor peso semántico. El valor de esta definidora indica que el dinero es una restricción fundamental para la conducta de consumo, y puntualiza la importancia de fortalecer las políticas públicas dirigidas a incrementar el costo monetario de la sustancia mediante los impuestos, así como su accesibilidad, vía la reglamentación y la vigilancia de su cumplimiento. Otras restricciones observadas son portar uniforme y estar en la escuela, datos que indican la importancia de mantener y fortalecer los reglamentos de las instituciones como un medio para evitar que disminuya el costo del consumo de alcohol.

La perspectiva metodológica usada mostró su utilidad para explorar otras dimensiones del consumo de drogas, en este caso, las restricciones al consumo de alcohol. En conjunto, los datos señalan la necesidad de asegurar e incrementar las sanciones asociadas con la venta de alcohol a menores e incluir estrategias para regular su disponibilidad, no sólo en el nivel comercial, sino también en ambientes sociales y familiares. Los resultados obtenidos pueden contribuir a fortalecer los programas preventivos, al mostrar algunas variables que incrementan la dificultad para que los adolescentes consuman alcohol.

\section{REFERENCIAS}

Amlung, M.T., Acker, J., Stojek, M.K., Murphy, J.G. \& MacKillop, J. (2012). Is talk "cheap"? An initial investigation of the equivalence of alcohol purchase task performance for hypothetical and actual rewards. Alcoholism: Clinical and Experimental Research, 36(4), 716-724.

Babor, T.F., Caetano, S., Casswell, G., Edwards, N., Giesbrecht, K., Graham et al. (2010). El alcohol: un producto de consumo no ordinario. Investigación y
En particular, las campañas dirigidas a prevenir y reducir el consumo en grupos vulnerables necesitan fundamentarse en sus motivaciones hacia el uso de sustancias (Cho, 2012). En este sentido, podría resultar efectivo diseñar las campañas de salud con mensajes basados en información recopilada mediante técnicas como las redes semánticas, debido a que permiten acceder a los significados y conocimientos socialmente compartidos en torno a un concepto.

Por último, entre las limitaciones del trabajo se destaca la naturaleza no probabilística de la muestra, lo que impide generalizar los resultados del estudio a otro grupo de adolescentes. Esta limitante, al mismo tiempo, señala la oportunidad para continuar trabajando en este campo, con muestras más amplias, además de explorar la utilidad de otras variables relevantes en este enfoque teórico, como variaciones en sus recursos (por ejemplo, ingreso o tiempo libre).

En la investigación sólo se exploraron las restricciones directas a la conducta de consumo; sin embargo, en el marco de la economía conductual se plantea la necesidad de modificar, a la par, las restricciones a las actividades gratificantes libres de drogas. Por lo tanto, es conveniente realizar estudios de análisis semántico para identificar los factores que pueden contribuir a reducir el costo de las actividades libres de consumo valiosas para el adolescente (actividades sociales, tiempo libre, etc.,), con el objetivo de aumentar la participación y el disfrute de los adolescentes en estas actividades (Murphy, Barnett \& Colby, 2006). •

políticas públicas. Washington, DC: Organización Panamericana de la Salud.

Bickel, W.K. \& Marsch, L.A. (2001). Toward a behavioral economic understanding of drug dependence: Delay discounting processes. Addiction, 96, 73-86.

Borges, G., Benjet, C., Medina-Mora, M.E., Orozco, R. \& Wang, P.S. (2008). Treatment of mental disorders for adolescents in Mexico City. Bulletin World Health Organization, 86, 757-764. 
Borsari, B., Murphy, J.G. \& Barnett, N.P. (2007). Predictors of alcohol use during the first year of college: Implications for prevention. Addictive Behaviors, 32(10), 2062-2086.

Celis, A. (2003). La salud de los adolescentes en cifras. Salud Pública de México, 45, 153-166.

Centros de Integración Juvenil. (2007). Estudio Básico de Comunidad Objetivo, 2007. México: CIJ, Dirección de Investigación y Enseñanza, Subdirección de Investigación.

Cho, H. (Ed.). (2012). Health Communications Message Design: Theory and Practice. Thousand Oaks, C.A: Sage.

Chung, T., Colby, S.M., Barnett, N.P. \& Monti, P.M. (2002). Alcohol Use Disorders Identification Test: Factor Structure in an Adolescent Emergency Department Sample. Alcoholism: Clinical and Experimental Research, 26(2), 223-231.

DeGrandpre, R.J., Bickel, W.K., Hughes, J.R., Layng, M.P. \& Badger, G. (1993). Unit price as a useful metric in analyzing effects of reinforcer magnitude. Journal of the Experimental Analysis of Behavior, 60, 641-666.

DeGranpre, R.J \& Bickel, W.K. (1996).Drug dependence as a consumer demand. En: L. Green \& J.H. Kagel (Eds.). Advances in behavioral economics (Vol.3). Substance use and abuse. Norwood, NJ: Ablex Publishing Co.

Del Boca, F.K., Darkes, J., Greenbaum, P.E. \& Goldman, M.S. (2004). Up close and personal: Temporal variability in the drinking of individual college students during their first year. Journal of Consulting and Clinical Psychology, 72, 155-164.

Díaz, L.R., Díaz, A., Hernández, C.A., Fernández, H., Solís, C. \& Narro, J. (2009). El consumo riesgoso y dañino de alcohol y sus factores predictivos en adolescentes estudiantes de bachillerato. Salud Mental, 32, 447-458.

Figueroa, J., González, E. \& Solís, V. (1981). Una aproximación al problema del significado: las redes semánticas. Revista Latinoamericana de Psicología, 13(3), 447-458.

Gentile, N.D., Librizzi, E.H. \& Martinetti, M.P. (2012). Academic constraints on alcohol consumption in college students: a behavioral economic analysis. Experimental and Clinical Psychopharmacology, 20(5), 390-399.
Guerrero, C.M., Muñoz, J.A., Sáenz, B., Pérez, R. \& Reynales, L.M. (2013). Impacto del consumo nocivo de alcohol en accidentes y enfermedades crónicas en México. Salud Pública de México, 55(2), 282-288.

Harrison, P.A., Fulkerson, J.A. \& Park E. (2000). The relative importance of social versus commercial sources in youth access to tobacco, alcohol, and other drugs. Preventive Medicine, 31, 39-48.

Instituto Nacional de Psiquiatría Ramón de la Fuente Muñiz; Instituto Nacional de Salud Pública; Secretaría de Salud (2012). Encuesta Nacional de Adicciones 2011: Reporte de Alcohol. En: Medina-Mora, M.E., Villatoro, J.A., Fleiz, C., Téllez, M.M., Mendoza, L.R., Romero, M., Gutiérrez, J.P., Castro, M., Hernández, M., Tena, C., Alvear, C. \& Guisa, V. México: INPRFM.

Kypri, K., Bell, M.L., Hay, G.C. \& Baxter, J. (2008). Alcohol outlet density and university student drinking: a national study. Addiction, 103, 1131-1138.

Medina-Mora, E., Carreño, S. \& De la Fuente, J. R. (1998). Experience with the alcohol use disorders identification test (AUDIT) in Mexico. Recent Developments in Alcoholism, 14, 383-396.

Medina-Mora, M.E., Robles, R., Cortina, D. \& Real. T. (Eds.). (2009). Evaluación de políticas públicas para el control del abuso de alcohol en México. México: Instituto Nacional de Psiquiatría, Secretaría de Salud. Medina-Mora, M.E., Villatoro, J.A, Fleiz, C., Téllez, M.M., Mendoza, L.R., Romero, M., Gutiérrez, J.P, Castro, M., Hernández, M., Tena, C., Alvear, C. \& Guisa, V. (2012). Encuesta Nacional de Adicciones 2011: Reporte de Alcohol. México: InPRFM.

Murphy, J.G. \& MacKillop, J. (2006). Relative reinforcing efficacy of alcohol among college student drinkers. Experimental and Clinical Psychopharmacology, 14, 219-227.

Murphy, J.G. Correia, C.J. \& Barnett, N.P. (2007). Behavioral economic approaches to reduce college student drinking. Addictive Behaviors, 32, 2573-2585.

Murphy, J.G., Barnett, N.P. \& Colby, S.M. (2006). Alcoholrelated and alcohol-free activity participation and enjoyment among college students: a behavioral theories of choice analysis. Experimental and Clinical Psychopharmacology, 14(3), 339-349.

Paschall, M.J., Grube, J.W., Black, C.A. \& Ringwalt, C.L. (2007).Is commercial alcohol availability related to 
adolescent alcohol sources and alcohol use? Findings from a multi-level study. Journal of Adolescent Health, $41,168-74$

Reyes-Lagunes \& Valdez, M. (1993). La construcción de instrumentos de medición a partir de Categorías de redes Semánticas. Un caso ilustrativo: el auto concepto. Revista de Psicología Social y Personalidad, 9(1), 57-66.

Rodríguez, J., Angulló, T. \& Angulló, M. (2003). Jóvenes, fin de semana y uso recreativo de drogas: evolución $\mathrm{y}$ tendencias del ocio juvenil. Adicciones: Monografía de drogas recreativas, 15(2), 7-34.

Saunders, J.B., Aasland, O.G., Babor, T.F., de la Fuente, J.R. \& Grant, M. (1993). Development of the Alcohol Use Disorders Identification Test (AUDIT): wHO collaborative project on early detection of persons with harmful alcohol consumption. II. Addiction, 88, 791804.

Scoppetta, O., Pérez, A. \& Lanziano, C. (2011). Perfiles asociados al consumo de alcohol de adolescentes escolarizados mediante análisis de correspondencias múltiples. Acta Colombiana de Psicología, 14(1), 139146.

Skidmore J.R. \& Murphy, J.G. (2011). The effect of drink price and next-day responsibilities on college student drinking: a behavioral economic analysis. Psychology of Addictive Behaviors, 25(1), 57-68.
Toumbourou, J.W, Stockwell, T., Neighbors, C., Marlatt, G.A., Sturge, J. \& Rehm J. (2007). Interventions to reduce harm associated with adolescent substance use. The Lancet, 369 (9570), 1391-1401.

Tremblay, P.F., Graham, K., Wells, S., Harris, R., Pulford, R. \& Roberts, S.E. (2010). When do first-year college students drink most during the academic year? An internet-based study of daily and weekly drinking. Journal of American College Health, 58(5), 401-411.

Vuchinich, R.E. \& Heather, N. (2003).Choice, behavioural economics, and addiction. Oxford, U.K.: Pergamon.

Vuchinich, R.E. \& Tucker, J.A. (1988). Contributions from behavioral theories of choice to an analysis of alcohol abuse. Journal of Abnormal Psychology, 97, 181-195.

Weitzman, E.R. \& Kawachi, I. (2000). Giving means receiving: the protective effect of social capital on binge drinking on college campuses. American Journal of Public Health, 90(12), 1936-1939.

Wood, P.K., Sher, K.J. \& Rutledge, P.C. (2007). College student alcohol consumption, day of the week, and class schedule. Alcoholism: Clinical and Experimental Research, 31(7), 1195-1207.

World Health Organization. (2011). Global status report on alcohol and health. Geneva, Switzerland: wHo Press. 